\title{
5p13 microduplication syndrome
}

INSERM

\section{Source}

INSERM. (1999). Orphanet: an online rare disease and orphan drug data base. 5 p13

microduplication syndrome. ORPHA:329802

5 p13 microduplication syndrome is a rare partial autosomal trisomy/tetrasomy

characterized by g lobal developmental delay, intellectual disability, autistic behavior, muscular hypotonia, macrocephaly and facial dysmorphism (frontal bossing, short palpebral fissures, low set, dysplastic ears, short or shallow philtrum, high arched or narrow palate, microg nathia). Other associated clinical features include sleep disturbances, seizures, aplasia/hypoplasia of the corpus callosum, skeletal abnormalities (large hands and feet, long fingers and toes, talipes). 